\section{COVID and consent}

Sir, prior to the COVID-19 pandemic, patients undergoing invasive procedures were subject to confirming their consent through written means, a process which is considered common within surgical fields. ${ }^{1}$ The guidance issued by the FGDP on 1 June suggested a move towards provision of 'digital packs' and it seems many standard operating procedures (SOPs) being issued by practices include the provision of digital information packs with consent forms to patients. ${ }^{2}$

Patients being issued consent forms to complete on their own may require additional support in order to ensure their validity so that consent is maintained and not overlooked, in light of the new working protocols the profession faces. Furthermore, in cases where patients are being triaged remotely, a complete exam may not be possible prior to execution of treatment: a key difference which has to be considered when exploring the options for consultation and delivery of clinical information. ${ }^{3}$

There is a risk due to patients' comprehension of the intended procedure and the lack of opportunity to fully appreciate or discuss the risks and benefits. Additionally, the change from a face-to-face consultation and 'cooling-off' period, as is the case with the gold-standard two stage consent, is likely to be affected. There is also a risk of clinicians not engaging with such measures which could be viewed as a noncompliant attitude to SOPs and guidelines, which although essential to good practice have come to be a burden to many.

\section{S. Stagnell, Winchester, R. Moore, Leeds}

\section{References}

1. Royal College of Surgeons of England. 3.5.1 Consent Available at: https://www.rcseng.ac.uk/standardsand-research/gsp/domain-3/3-5-1-consent/ (accessed June 2020).

2. FGDP(UK). Implications of COVID-19 for the safe management of general dental practice - a practical guide. Available at: https://www.fgdp.org.uk/ implications-covid-19-safe-management-generaldental-practice-practical-guide (accessed June 2020).

3. General Medical Council. Remote consultations. Available at: https://www.gmc-uk.org/ethicalguidance/ethical-hub/remote-consultations (accessed June 2020)

$$
\text { https://doi.org/10.1038/s41415-020-1903-1 }
$$

\section{No chisels}

Sir, in reference to the correspondence entitled Altered exodontia techniques (BDJ 2020; 228: 811-812) advocating chisels and mallets in order to avoid aerosol generating procedures (AGP), a practical alternative is utilising a straight handpiece with a straight fissure bur and non-simultaneous irrigation. This would not qualify as an AGP and would be safer and more psychologically tolerable to patients as well. ${ }^{1}$

\section{Sahni, New Delhi, India}

\section{Reference}

1. Hammond D, Hughes F, Stirrup P, Barkworth N. Setting up and maximising the usage of an Urgent Dental Care Centre in Blackpool. Sharing our experiences. Br J Oral Maxillofac Surg 2020 doi: 10.1016/j.bjoms.2020.05.008 [Epub ahead of print].

https://doi.org/10.1038/s41415-020-1904-0

\section{SDF - a game changer?}

Sir, current guidance from the RCS

Recommendations for paediatric dentistry during the COVID-19 pandemic is very clear: 'Access to general anaesthesia will be significantly reduced for the foreseeable future. ${ }^{1}$ As a foundation dentist, I have had little exposure to silver diamine fluoride (SDF) during my undergraduate studies and so was very interested by the recent $B D J$ paper highlighting its use in the management of dental caries. ${ }^{2}$ Through the utilisation of careful non-AGP application methods, it can prevent the progression of carious lesions and arrest them entirely although the most immediate issue is that it is currently not licensed to be used as a caries-arresting agent in the UK.

SDF has huge potential within community dentistry and general dental practices, more so at this unprecedented time. This would be particularly useful in coping with the reduced GA access and managing extensive carious lesions atraumatically, particularly in uncooperative patients. Considering all of the benefits, it begs the following questions - why is it still unlicensed as a caries-arresting agent in the UK? Where is the official guidance on its use? And finally, why has it not become a prevalent form of treatment considering its efficacy and appropriateness in the current climate?

\section{A. Remtulla, Bedford, UK}

\section{References}

1. Royal College of Surgeons of England. Recommendations for paediatric dentistry during the COVID-19 pandemic. 2020. Available at: https:// www.rcseng.ac.uk/-/media/files/rcs/fds/guidelines/ paediatric-dentistry-covid19.pdf (accessed June 2020).

2. Greenwall-Cohen J, Greenwall L, Barry S. Silver diamine fluoride - an overview of the literature and current clinical techniques. Br Dent J 2020; 228: 831-838. https://doi.org/10.1038/s41415-020-1905-z

\section{Sedation during the pandemic}

Sir, the COVID-19 pandemic has seen the drawback of much needed pharmacological behaviour management services (ie IV sedation, IHS and GA). In experiencing such difficulties in the UDC hubs, I have become a huge proponent of the often underused and overlooked use of oral sedation techniques in the management of dentally anxious patients who have previously relied on IHS or IV sedation. Via anecdotal feedback, many patients are realising that they would be content to accept future treatment under such measures.

With dental practices now resuming a limited level of practice and possibly experiencing the level of frustrations with lack of treatment provisions for anxious patients, oral sedation with Diazepam is an invaluable tool in enabling patients the access to care they require. Increasing confidence in the use of Diazepam pre-medication is paramount for the changing face of dentistry, especially for those of us who have, in the past, become overly reliant on the GA, especially in polypharmacy patients. Careful case selection is of course key to its successful use, and requires the triaging clinician to be thorough in ascertaining dental history and indication of sedation need.

Appropriate prescription of Diazepam prior to attendance for urgent dental care can create positive outcomes for both the patient and treating clinician and just may result in a cultural shift that reduces the burden on sedation services when normal service resumes.

G. Atkinson, Salford, UK https://doi.org/10.1038/s41415-020-1906-y

\section{Dental dam: the time is right}

Sir, dental/rubber dam has always been a strange issue in dental practice. Those of us who use it 'swear by it', but those who don't, decry its use, often in most strident terms.

There is absolutely no doubt that one of the major advantages of using it is the reduction in bacterial (viral) aerosol. Given the difficult and troubled time we now work under we must assume that dental dam could be a life saver, and it would seem to be professionally 